\title{
Instruments of active search and assessment of memory complaint. a systematic review
}

\begin{abstract}
Introduction: promote an active search for memory complaint states as a means of predicting neuropsychological symptoms in order to propose an initial adequate intervention.

Objective: identify the instruments that address active search and memory complaint and the context in which they were used.

Metodology: systematic literature review conducted in Pubmed-Medline, Lilacs, Scielo, Web Of Science, Scopus, CAPES thesis database and PsycINFO.

Results: 2227 articles were located; the final sample consisted of 27 articles selected by abstracts and after full reading. The analysis allowed identification of 15 instruments. Of the active memory complaint search instruments, $18,6 \%$ corresponded to MAC-Q Questionnaire, $14,8 \%$ to MFE, $11,1 \%$ to PRMQ, $11,1 \%$ to McNair, $7,4 \%$ to MAC-S and other questionnaires represented $3,7 \%$ each.
\end{abstract}

Conclusion: the instruments pose as a fundamental tool for memory complaint tracking and have been being utilized in the most diverse research scopes.

Keywords: memory complaint, memory, instrument

\author{
Volume 7 Issue 2 - 2017
}

\author{
Estela Barbosa Ribeiro,' Mariélli Terassi,' \\ Estefani Serafim Rossetti,' Francisco Assis \\ Carvalho Vale ${ }^{2}$ \\ 'Universidade Federal de São Carlos- UFSCar - São Carlos (SP), \\ Brazil \\ ${ }^{2}$ Department of Medicine, Universidade Federal de São Carlos - \\ UFSCar - São Carlos (SP), Brazil
}

\begin{abstract}
Correspondence: Estela Barbosa Ribeiro, Gerontologist Master's student in Nursery for the Nursery Post-graduation Programme at Universidade Federal de São Carlos - UFSCar -
\end{abstract} São Carlos (SP), Brazil, Email estelabribeiro@gmail.com

Received: June 27, 2017| Published: July 25, 2017

\section{Introduction}

The older adults population is increasing at a fast rate around the globe. Aging is accompanied by cognitive skills decrease, memory being one of them. The ability to create, retain and use memory is an essential property in individual's survival. ${ }^{1}$ Older adults often complaint about mnemonic impairment, although it doesn't pose as pathological in some cases. ${ }^{2}$

According to Yassuda et al. ${ }^{3}$ diverse hypothesis have been formulated over the past few years to explain memory performance decrease in older adults population. The most recent refers to the idea that attitudes and beliefs interfere negatively in memory. The set of ideas and feelings about memory is known in the literature as meta-memory. ${ }^{3}$ Some authors state that memory complaints can foreshadow dementia outcomes in older adults. ${ }^{2}$ Studies highlight the importance of memory complaint in high schooling older adults for early detection of cognitive impairment, due to its non-identification in brief assessments. ${ }^{2,4,5}$

Memory loss is a frequent complaint for older adults in medical appointments, being reported for $25 \%-50 \%$ of these individuals. ${ }^{6}$ Estimates say the prevalence of memory loss corresponds to $46,3 \%$ in adults from 50 to 59 years of age and $63,4 \%$ for adults from 80 to 100 years of age, ${ }^{7}$ Being a female and having low schooling level have also been associated with highest prevalence of memory loss. ${ }^{7}$ Memory loss due to aging is associated not only to anatomicalphysiological alterations, but to cognitive exercise reduction in the learning process as well. ${ }^{5,8}$ Older adults who are active in learning processes show favorable intellectual and cognitive performance when compared to non-active older adults. ${ }^{5}$ Reasoning exercises, new information acquisition and daily reading contribute to keep older adults active..$^{5,9}$

There are two techniques described in the literature to assess memory loss. Questionnaires with experimental tasks that aim to estimate the recordation probability of previously learned information, ${ }^{10}$ and self-reported instruments that address the experienced memory issues. ${ }^{11}$ Researches evidence that subjective memory assessments present difficulties regarding real patient's performance, making it difficult to measure memory issues via objective assessment tests. ${ }^{12}$ This assessment hardship is associated to the discretion of the tests, the requisition of specialized professionals and the long periods of evaluations. $^{12}$

On that aspect, this systematic review aimed to identify the active memory complaint search instruments and the context in which they were used. The reason relies in addressing the importance of acknowledging the active memory search instruments by health professionals, since these instruments can help provide an early diagnosis of dementias in older adults, including Alzheimer's and depression, thus allowing means to a qualified assistance.

\section{Methodology}

\section{Search strategy}

Searching occurred during two months - April and May 2014. Databases utilized were Pubmed-Medline, Lilacs, Scielo, Web of Science, Scopus, CAPES thesis database, and PsycINFO, entering the following descriptors: "subjective memory complaint", "memory complaint", "questionnaire", "scale", "instrument" e "assessment". The combinations used in the searching were as follows: "subjective memory complaint AND assessment", "memory complaint AND assessment", "subjective memory complaint AND instrument", "memory complaint AND instrument", "subjective memory complaint AND questionnaire", "memory complaint AND questionnaire", "subjective memory complaint AND scale" and "memory complaint AND scale". Articles published from 2009 to 2014 were considered.

\section{Identification and screening}

The criteria adopted for this review included:(1)English, Portuguese or Spanish language; (2) Articles/papers, dissertations and thesis.

Articles selection was blind and independently conducted by two groups of evaluators, and divided into in two steps: 1 . Selection by 
titles and abstracts; 2. Full article reading. Afterwards the groups gathered to assess possible divergences and find consensus according to PRISMA protocol.

The Preferred Reporting Items for Systematic Reviews and Metaanalyses (PRISMA) protocol - presented in Figure 1 - was proposed by Moher et al., ${ }^{13}$ to help authors improve communication of systematic reviews and meta-analyses besides using for critical evaluations of published reviews. The duplicated articles exclusion process utilized the Minilist app*.
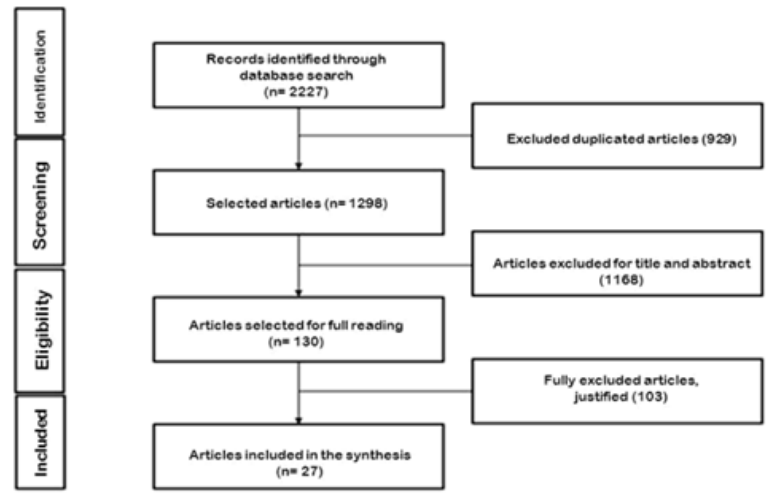

Figure I Identification and selection of found articles, according to PRISMA protocol: Source Moher et al., ${ }^{13}$

\section{Analysis and selection strategies \\ Eligibility criteria}

The articles that did not use active search instruments to classify memory complaint were excluded. Three of the found articles utilized questions from validated instruments, but were excluded for not utilizing the instrument as a whole. Two of the found instruments did not assess memory complaint. However, the studies utilized the instruments to characterize their subjects as complainants. Being so, these studies and instruments were considered compatible with the purpose of this paper and gained inclusion in the review.

\section{Results}

There were found 2227 articles, of which 929 were excluded for being duplicates. The next step consisted of the exclusion of 1298 articles based on their title or abstract. After these procedures, there were 130 articles left for full reading, of which 103 were excluded for not being pertinent.

The studies selected for the review counted 27, all of them being scientific paper (article) type publications. A total of 15 instruments were found in those articles (Table 1). *App designed to the referred end, by Rafael Campanari (2014). To result analysis end, the articles were grouped by instruments as shown in Table 1. Of the found studies, 22,2\% were conducted in Spain, 22,2\% in Brazil, 14,9\% in the USA, $11,1 \%$ in Australia, $11,1 \%$ in France, $3,7 \%$ in Holland, $3,7 \%$ in Canada, 3,7\% in Sweden, 3,7\% in Switzerland and 3,7\% in Germany. The following instruments were identified: Memory Complaint Questionnaire (MAC-Q); Memory Failures in Everyday Memory (MFE); Questionnaire for Subjective Memory Complaints (QSMC) - short version; Memory Complaint Scale (MCS); Prospective and Retrospective Daily Memory Failure Questionnaire (PRMQ); Multifactorial Memory Questionnaire (MMQ); McNair Frequency of Forgetting Questionnaire (McNair); Memory Assessment ClinicsSelf-Rating Scale (MAC-S); The Measure of Insight into Cognition - Self-Report (MIC-RS); Corsi Block-Tapping Test (CBT); Hopkins
Verbal Learning Test-Revised (HVLT-R); Forgetting Frequency Scale; Evaluation of Balance and Memory Problems (EVATEM); Meta-memory In Adulthood Questionnaire (MIA) and Memory Functioning Questionnaire (MFQ).

Through results analysis it was possible to identify the dominance of the MAC-Q instrument corresponding to $18,6 \%$ of the studies, followed by $4,8 \%$ of MFE , $11,1 \%$ of PRMQ, $11,1 \%$ of McNair, 7,4\% of MAC-S and 3,7\% each of the others. The design of the instruments is presented next.

\section{Memory Complaint Questionnaire (MAC-Q)}

Composed of six questions that assess memory complaint in five daily situations, with five different possible answers; scores higher or equal to 25 points are considered indicative of subjective memory compromising, and the maximum punctuation stands for 30 points. ${ }^{14}$

\section{Memory failures of everyday questionnaire (MFE)}

Utilized to assess every day forgetting; it contains 28 items regarding daily life situations and activities with nine possible answers to each item. ${ }^{15}$

\section{Questionnaire for subjective memory complaints (QSMC) - short version}

Brief, reliable and validated questionnaire used to assess memory complaints and to screen for dementia. ${ }^{16}$

\section{Memory complaint scale (MCS)}

Used for memory complaint identification also being useful to differentiate demented from normal older adults. It is composed of seven question, where: No MC (0-2), slight MC (3-6), mild MC (710) and accentuated MC (11-14). ${ }^{17}$

\section{Prospective and retrospective daily memory failure questionnaire (PRMQ)}

Composed of 16 questions, it assesses subjective memory. Participants classify how many times each event happened using a five-point scale, ranging from (1) never to (5) very often. ${ }^{18}$

\section{Multifactorial memory questionnaire (MMQ)}

It assesses three dimensions of self-reported memory, including general satisfaction and satisfaction with self-memory capacity (MMQ-contentment), every day memory capacity perception (MMQ-capacity) and the use of every day memory strategies (MMQstrategy). ${ }^{19}$

\section{McNair frequency of forgetting questionnaire (McNair)}

Participants indicate how often they forget words, people's names, among other items commonly forgotten punctuating: (0) Never, (1) Sometimes, (2) Frequently, (3) Always. The instrument punctuation score ranges from 0 to 45 points, with higher scores indicating more frequent memory complaints or forgotten items. None of the studies presented a cut point for the frequency of forgotten items, which would be an indication of clinically significant memory deficits. ${ }^{20}$

\section{Memory assessment clinics-self-rating scale (MAC-S)}

Consisted of 45 questions divided into two scales: abilities (21 items), subdivided into numerical recordation, daily tasks, remote personal memory, semantic memory; and occurrence frequency (24 itens), subdivided into semantic memory, attention/concentration, guided memory, general forgetting, facial recognition. ${ }^{21}$ 


\section{The measure of insight into cognition - self-report (MIC-RS)}

Utilized to assess the report of subjective cognitive complaints in attention, executive functioning and memory. It evaluates difficulty frequency in 12 items, generating a composed score from 0 to 36 . The highest punctuation indicates more frequent neuropsychological problems. ${ }^{22}$

\section{Corsi block-tapping test (CBT)}

Assesses attention and memory, particularly attention amplitude and work memory performance. The test consists of a plaque with randomly distributed cubes on it, and is divided into two parts: at first the examiner observes cubes after a predetermined sequence and the assessed subject must reproduce the sequence in the same order; next, the subject must execute the presented sequence without reversing it. The score varies from 0 to 9 points. ${ }^{23}$

\section{Hopkins verbal learning test - revised (HVLT-R)}

Brief learning and verbal memory test with six alternative forms. Ideally used for situations requiring neuropsychological examinations and assessment of abnormal forgetting. ${ }^{24}$

\section{Forgetting frequency scale}

The definitive version of the questionnaire includes four sheets and accompanies two other supplementary sheets: first is the introduction sheet referring to the existence of daily memory lapses experiences including the forgetting frequency scale points one to seven meanings. ${ }^{25}$

\section{Evaluation of balance and memory problems (EVATEM)}

It prospectively evaluates the predictive test values for diagnosis of slight cognitive problems and Alzheimer's disease in $65+$ older adults. If a memory complaint is identified (through a specific questionnaire), the five words test, cognitive disturb examination test and verbal fluency test are applied. ${ }^{26}$

\section{Meta-memory in adulthood questionnaire (MIA)}

It contains 108 questions about individual general memory knowledge, memory processes and tasks divided into seven scales: memory strategies use, knowledge of basic memory processes, anxiety or effort sentiment related to memory performance, awareness of memory changes with age, memory capacity knowledge, and personal control of the ability to recollect. ${ }^{27}$

\section{Memory functioning questionnaire (MFQ)}

Composed of 64 items divided into four scales: general forgetting frequency, severity of forgetting, retrospective functioning and mnemonic use. This instrument does not distinguish prospective memory items. ${ }^{28}$

\section{Discussion}

Of the instruments found in the selected studies shown in Table 1, only the most important will be addressed here. Studies that classified their subjects as complainants or non-complainants using non-systematic questions from particular instruments, and those which utilized clinical assessments or multidimensional instruments will not be addressed, for they do not refer to a specific instrument, rather to open questions regarding active memory complaint search as in Bartley et al. ${ }^{29}$ the study used questions such as the following to assess memory complaint: Do you think you have memory problems? Do you have difficulties remembering conversations?

Morga and Steven, ${ }^{30} \&$ Lima-Silva and Yassuda ${ }^{25}$ used the Memory Complaint Questionnaire (MAC-Q) in their researches addressing $55+$ older adults but no significant results were obtained regarding memory complaint (MC). However, Buckley et al., ${ }^{31}$ addressed a population of $111260+$ older adults, aiming to calculate the transition rates from older adults that were healthy (HOS) to Mild Cognitive Impairment (MCI). Results demonstrated that $\mathrm{MC}$ was more severe in MCI patients than in the HOS group. Within the HOS group MC severity was related only to affective variables, whilst in the MCI group it was related only to age. As in Buckley et al., ${ }^{31}$ the authors Brum et al., ${ }^{20}$ investigated general memory assessment and strategy use among MCI older adults using MAC-Q and McNair Frequency of Forgetting Questionnaire instruments. The MCI participants did not report greater change and forgetting frequencies perception. These findings suggest $\mathrm{MCI}$ older adults do not inform precisely or do not perceive their deficits.

McNair questionnaire was also used in Wafta et al., ${ }^{32}$ and KearneySchwartz et al., ${ }^{33}$ studies, both of which addressed hypertensive older adults with MC investigating anti-hypertensive drug action on cognition. As a result, the studies showed that using drugs from the calcium channel blockers class was associated with better cognitive performance, regardless of blood pressure levels, usual cardiovascular risk factors and vessel structure, suggesting a protector effect from this pharmacological class.

Ronnlund et al., ${ }^{34}$ examined self-reported daily memory failure predictors using the Prospective and Retrospective Daily Memory Failure Questionnaire (PRMQ) in an older adults population with memory complaints. Results did not evidence relation between the instrument and objective memory. Taken together, these findings are indicative more of a reflection of humor states and personality regarding self-reported memory assessed by PRMQ in an older population than individual differences in memory and cognitive capacity. Eschen et al., ${ }^{35}$ and Steinberg et al., ${ }^{36}$ assessed PRMQ utility for an initial MCI screening; amongst its findings MCI patients reported greater prospective and retrospective memory deficits. In a healthy older adults sample, the authors described a higher quantity of subjective prospective memory complaints compared to retrospective memory.

Montejo et al., ${ }^{37}$ examined the Memory Failures of Everyday (MFE) instrument structure in 19 to 64 year-old participants. Results supported the existence of a structure in the three-factor instrument (do activities, communication monitoring, and recognition) explaining $29,3 \%$ of the variance. The first two factors presented high positive correlation between them and a higher association with humor and memory generated complaints. On the other hand, recognition displayed higher positive correlation to age and negative correlation to objective performance memory. García-Sevilla et al., ${ }^{23}$ also utilized MFE to assess MC performance in individuals graduated from the memory training programme at Murcia University, Spain. Montejo et al., ${ }^{38}$ evaluated the association between $\mathrm{MC}$ and health, humor and episodic memory perception. These authors state that a more structured tool like MFE would be strongly correlated to other variables in comparison to non-structured memory complaint open questions (OQ). Based on the findings, MFE appears to be a more precise measure than $\mathrm{OQ}$ in detecting MCI predictors.

Vale et al., ${ }^{17}$ proposed the Memory Complaints Scale structured questionnaire as an instrument to active memory complaint search in order to investigate its utility to identify dementia in cognitively 
healthy older adults. Through a random sample extracted from a specialized teaching hospital casuistry, $60+$ older adults were selected.

The coefficients found for the scale demonstrated to be reliable indicating good internal data consistency and informative properties to the under investigation construction.

Table I Studies characteristics and instruments

\begin{tabular}{|c|c|c|c|c|}
\hline Authors/Year & Country & Main objective (MC Related to) & Age (In Years) & Type of Study Cut \\
\hline \multicolumn{5}{|c|}{ Memory Complaint Questionnaire (MAC-Q) } \\
\hline Lima-Silva, Yassuda/2009 25 & Brazil & Cognitive performance & $56-92$ & Transversal \\
\hline Morga, John Stevens/2010 30 & Australia & Herbal medicine & $\geq 55$ & Transversal \\
\hline R Buckley et al./2013 $3^{31}$ & Australia & Comparison with AD & $\geq 60$ & Transversal \\
\hline Brum et al. $/ 2013^{20}$ & Brazil & Comparison with $\mathrm{MCl}$ & $\geq 60$ & Transversal \\
\hline Porto et al./2013 & Brazil & Cognitive tests assessment & $>60$ & Transversal \\
\hline \multicolumn{5}{|c|}{ Memory Failures in Everyday Memory (MFE) } \\
\hline García-Sevilla et al./2014 ${ }^{23}$ & Spain & Effects of training types & $61-81$ & Transversal \\
\hline Montejo et al./2014 ${ }^{37}$ & Spain & Correlation between humor, episodic memory and health & $65-87$ & Transversal \\
\hline Montejo et al./2014 ${ }^{38}$ & Spain & Humor e anxiety & $19-60$ & Transversal \\
\hline Montejo-Carrasco et al./ 20II & Spain & Clinical assessment & $65-89$ & Transversal \\
\hline \multicolumn{5}{|c|}{ Questionnaire for Subjective Memory Complaints (QSMC) - short version } \\
\hline Juncos-Rabadan et al./20I2 & Spain & Cognitive impairment & $\geq 50$ & Transversal \\
\hline \multicolumn{5}{|l|}{ Memory Complaint Scale (MCS) } \\
\hline Vale et $\mathrm{al} / 2012^{17}$ & Brazil & Questionnaire development & $\geq 60$ & Transversal \\
\hline \multicolumn{5}{|c|}{ Prospective and Retrospective Daily Memory Failure Questionnaire (PRMQ) } \\
\hline Eschen et al./2009 ${ }^{35}$ & Switzerland & Initial screening for $\mathrm{MCl}$ & $54-91$ & Transversal \\
\hline Ronnlund et al./20II ${ }^{34}$ & Sweden & Prevention fundaments & $60-90$ & Transversal \\
\hline Steinberg et al./ $2013^{36}$ & USA & Cognitive performance and psychological factors & $\geq 65$ & Transversal \\
\hline \multicolumn{5}{|c|}{ Multifactorial Memory Questionnaire (MMQ) } \\
\hline Lenehan et al./ $2012^{19}$ & Australia & Subtypes of memory complaint & $60-90$ & Transversal \\
\hline \multicolumn{5}{|c|}{ McNair Frequency of Forgetting Questionnaire (McNair) } \\
\hline Brum et al./ $2013^{20}$ & Brazil & Comparison with $\mathrm{MCl}$ & $\geq 60$ & Transversal \\
\hline Watfa et al./2010 32 & France & Pharmacological treatment and & $60-85$ & Transversal \\
\hline Kearney-Schwartz et al./2009 ${ }^{33}$ & France & cognitive performance & $60-85$ & Transversal \\
\hline \multicolumn{5}{|c|}{ Memory Assessment Clinics-Self-Rating Scale(MAC-S) } \\
\hline Adhikari et al./ $2011{ }^{39}$ & Canada & Depressive symptoms & $\geq 16$ & Transversal \\
\hline Ann Pearman/ 200940 & USA & Personality, depression and health & $19-28$ & \\
\hline \multicolumn{5}{|c|}{ The Measure of Insight into Cognition - Self-Report (MIC-RS) } \\
\hline Gooding et al./ $2012^{22}$ & USA & Cognitive rehabilitation for schizophrenics & \pm 40 & Transversal \\
\hline \multicolumn{5}{|l|}{ Block-Tapping Test (CBT) } \\
\hline García-Sevilla et al./2014 ${ }^{23}$ & Spain & Effects of cognitive training & $61-81$ & Transversal \\
\hline \multicolumn{5}{|c|}{ Hopkins Verbal Learning Test-Revised (HVLT-R) } \\
\hline Parker et al./2010 & USA & Brain function & $\geq 65$ & Transversal \\
\hline \multicolumn{5}{|c|}{ Escala de Frequência de Esquecimentos } \\
\hline Lima-Silva, Yassuda/2009 25 & Brazil & Cognitive performance & $56-92$ & Transversal \\
\hline \multicolumn{5}{|c|}{ Evaluation of Balance and Memory Problems (EVATEM) } \\
\hline Vannier-Nitenberg et al./2013 26 & France & Cognitive tests and falls & $\geq 65$ & Transversal \\
\hline \multicolumn{5}{|c|}{ Meta-memory in Adulthood Questionnaire(MIA) } \\
\hline Visser et al./ 2012 & Holland & Instrument development & \pm 48 & Transversal \\
\hline \multicolumn{5}{|c|}{ Memory Functioning Questionnaire (MFQ) } \\
\hline KatjaWerheid et al./ 2010 & Germany & Cognitive performance & \pm 66 & Transversal \\
\hline
\end{tabular}

Adhikari et al., ${ }^{39}$ used the Memory Assessment Clinics SelfRating Scale (MAC-S) instrument to assess MC in a group of Acute Respiratory Distress Syndrome (ARDS) survivors released from ICU after five years. The instrument assesses subjective evaluations of memory capacity in diverse personal situations. The MAC-S frequency factor is a subjective classification of memory problems occurrence with high test-retest reliability and concurrent validity. As the same, Pearman ${ }^{40}$ examined the role of several variables in his study with the MAC-S instrument, including personality, depression and health for both objective and subjective memory in young adults from 19 to 28 years old with primary findings being that health problems can affect many areas of healthy functioning including sleep, medicines use and energy, what may directly affect memory functioning. Furthermore, health problems might need constant monitoring changing into the source of anxiety increase and attention dispersion. Therefore, health should be considered an important source of information when memory is assessed and memory problems are reported throughout life.

\section{Conclusion}

This review brought evidences that despite the relative variety concerning age and population in the studies here addressed, the findings suggest that realizing an active memory complaint search is a means to predicting neuropsychological symptoms in order to provide initial adequate intervention. Although there is a consensus on properly developed and validated active memory complaint 
search instruments and their importance as a tool for clinical practice, they should be used carefully, always respecting construction and validation guidelines addressed by the authors and practice guidelines utilized by expert professionals.

\section{Acknowledgements}

Juliana Cerqueira Leite, Mariana Luciano de Almeida, Aryanne Pereira Soares Avalhai, Catia Cristina Thomaz, Keila Francisco Neto de Oliveira, Beatriz Rodrigues de Souza Mel, Zilda Rezende Mota Botan, Daniela Dalpubel, Nathalia Alves de Oliveira, Érica Mestor Souza, Gabriela Dutra Gesualdo and CAPES for financial support.

\section{Conflicts of interest}

None.

\section{Funding}

None.

\section{References}

1. Bruel-Jungerman E, Davis S, Laroche S. Brain Plasticity Mechanisms and Memory: A Party of Four. Neuroscientist. 2007;13(5):492-505.

2. Paulo DLV, Yassuda MS. Queixas de memória de idosos e sua relação com escolaridade, desempenho cognitivo e sintomas de depressão e ansiedade. Ver Psiq Clín. 2010;37(1):23-26.

3. Yassuda MS, Lasca VB, Neri AL. Meta-memória e auto-eficácia: um estudo de validação de instrumentos de pesquisa sobre memória e envelhecimento. Psicol Reflex Crít. 2005; 18(1): 78-90.

4. Costa DP. Avaliação de queixas de memória por meio do questionário de memória prospectiva e retrospectiva (PRMQ) numa amostra populacional na cidade de São Paulo. Tese Mestrado. Escola Paulista de Medicina, título de Mestra em Ciências. São Paulo, Brazil. 2010.

5. Park DC, Gutchess AH, Meade ML, et al. Improving Cognitive Function in Older Adults: Nontraditional Approaches. JJ Gerontol B Psychol Sci Soc Sci 62 spec no. 2007;1:45-52.

6. Jonker C, Geerlings MI, Schmand B. Are memory complaints predictive for dementia? A review of clinical and population- based studies. Int $J$ Geriatr Psychiatry. 2000;15(11):983-991.

7. Westoby CJ, Mallen CD, Thomas E. Cognitive complaints in a general population of older adults: prevalence, association with pain and the influence of concurrent affective disorders. Eur J Pain. 2009;13(9):970-976.

8. Neves VMS. Programa para a estimulação da memória de trabalho na terceira idade: abordagens modernas de treinamento. Dissertation presented to the Phonoaudiology post-graduation course at Universidade Veiga de Almeida as partial requirements for obtaining the Master Degree. Rio de Janeiro, Brazil. 2009.

9. Erickson K, Kramer AF. Aerobic exercise effects on cognitive and neural plasticity in older adults. Br J Sports Med. 2009;43(1):22-24

10. Chua EF, Schacter DL, Sperling RA. Neural correlates of metamory: a comparison of feeling -of-knowwing and retrospective confidence judgments. J Cogn Neurosci. 2009;21(9):1751-1765.

11. Jonker C, Smits CHM e Deeg DJH. Affect Relatedmeta-memory and memory performance in a population - based sample of older adults. Educational Gerontology. 1997;23(2):115-128.

12. Costa DP. Avaliação de queixas de memória por meio do questionário de memória prospectiva e retrospectiva (QMPR) numa amostra populacional na cidade de São Paulo. Master's Thesis. Escola Paulista de Medicina, Master in Sciences title. São Paulo, Brazil. 2010.
13. Moher D, Liberati A, Tetzlaff J, et al. Preferred Reporting Items for Systematic Reviews and Meta-Analyses: The PRISMA Statement. Annals of Internal Medicine. 2010;151(4):264-269.

14. Mattos P, Lino P, Rizo L, et al. Memory complaints and test performance in healthy elderly persons. Arq Neuro-Psiquiatr. 2003;61(4):920-924.

15. Carrasco PM, Peña MM, Sueiro MJ. The Memory Failures of Everyday Questionnaire (MFE): Internal Consistency and Reliability. Span $J$ Psychol. 2012;15(3):1424-1431.

16. Youn JC, Kim KW, Lee DY, et al. Development of the Subjective Memory Complaints Questionnaire. Dement Geriatr Cogn Disord. 2009;27(4):310-317.

17. Vale FAC, Balieiro-Jr AP, Silva-Filho JH. Memory complaint scale (MCS): Proposed tool for active systematic. Dementia \& Neuropsychologia. 2012;6(4).

18. Santos AT, Leyendecker DD, Costa ALS, et al. Subjective Memory Complaints, Cognitive Performance, and Psychological Factors in Healthy Older Adults. Rev Esc Enferm USP. 2012; 46(Esp):24-29.

19. Lenehan ME, Klekociuk SZ, Summers MJ. Absence of a relationship between subjective memory complaint and objective memory impairment in mild cognitive impairment (MCI): is it time to abandon subjective memory complaint as an MCI diagnostic criterion? Int Psychogeriatr. 2012; 24(9):1505-1514

20. Brum PS, Yassuda MS, Forlenza OV. Subjective memory and strategy use in mild cognitive impairment and healthy aging. Psychology \& Neuroscience. 2013;6(1):89-94.

21. Crook TH, Larrabee GJ. A self-Rating scale for evaluating de Vellis. Scale Development:theory and applications. Applied social research methods series. Sage publications, London, UK. 2003.

22. Gooding AL, Saperstein A, Mindt MR, et al. Predictors of treatment utilisation at cognitive remediation groups for schizophrenia: The roles of neuropsychological, psychological and clinical variables. Neuropsychological Rehabilitation. 2012;22(4):516-531.

23. García-Sevilla J, Fernández PJ, Fuentes LJ, et al. Estudio comparativo de dos programas de entrenamiento de la memoria en personas mayores conquejas subjetivas de memoria: um análisis preliminar. Anales de psicologia. 2014;30(1):337-345.

24. Benedict RHB, Schretlen D, Groninger L, et al. Hopkins Verbal Learning Test - Revised: Normative Data and Analysis of Inter-Form and TestRetest Reliability. The Clinical Neuropsychologist. 1998;12(1):43-55.

25. Lima-Silva TB, Yassuda MS. The relationship between memory complaints and age in normal aging. Dement Neuropsychol. 2009;3(2):94-100.

26. Vannier-Nitenberg C, Dauphinot V, Bongue B, et al. Early detection of memory impairment in people over 65 years old consulting at Health Examination Centers for the French health insurance: the EVATEM protocol. BMC Geriatrics. 2013;13:55.

27. Dixon RA, Hultsch DF, Hertzog C. The Meta-memory In Adulthood (MIA) questionnaire. Psychopharmacology Bulletin. 1988;24(4):671-688.

28. Gilewski MJ, Zelinski EM, Schaie KW. The Memory Functioning Questionnaire for Assessment of Memory Complaints in Adulthood and Old Age. Psychology and Aging. 1990;5(4):482-490.

29. Bartley M, Bokde, Ewers M, et al. Subjective memory complaints in community dwelling healthy older people: the influence of brain and psychopathology. Int J Geriatr Psychiatry. 2012;27(8):836-843.

30. Morga A, Stevens J. Does Bacopa monnieri Improve Memory Performance in Older Persons? Results of a Randomized, PlaceboControlled, Double-Blind Trial. J Altern Complement Med. 2010;16(7):753-759. 
31. Buckley R, Saling MM, Ames D, et al. Factors affecting subjective memory complaints in the AIBL biomarkers, memory, affect, and age. Int Psychogeriatr. 2013;25(8):1307-1315.

32. Watfa G, Rossignol P, Kearney-Schwartz A, et al. Use of calcium channel blockers is associated with better cognitive performance in older hypertensive patients with subjective memory complaints. J Hypertens. 2010;28(12):2485-2493

33. Kearney-Schwartz A, Rossignol P, Bracard S, et al. Vascular Structure and Function Is Correlated to Cognitive Performance and White Matter Hyperintensities in Older Hypertensive Patients With Subjective Memory Complaints. Stroke. 2009;40(4):1229-1236.

34. Ronnlund M, Vestergren P, Mantyla T, et al. Predictors of Self-Reported Prospective and Retrospective Memory in a Population-Based Sample of Older Adults. J Genet Psychol. 2011;172(3):266-284.

35. Eschen A, Martin M, Gasser US, et al. Prospective and Retrospective Memory Complaints in Mild Cognitive Impairment and Mild Alzheimer's Disease. Brain Impairment . 2009;10(1):59-75.
36. Steinberg SI, Negash S, Sammel MD, et al. Subjective Memory Complaints, Cognitive Performance, and Psychological Factors in Healthy Older Adults. AM J Alzheimers Dis Other Demen. $2013 ; 28(8): 776-783$

37. Montejo P, Montenegro M, Sueiro-Abad MJ, et al. Cuestionario de Fallos de Memoria de la Vida Cotidiana (MFE). Análisis de factores conpoblación española. Anales de psicologia. 2014;30(1): 320-328.

38. Montejo P, Montenegro M, Fernandez-Blazquez MA, et al. Association of perceived health and depression with older adults' subjective memory complaints: contrasting a specific questionnaire with general complaints questions. Eur J Ageing. 2014;11(1):77-87.

39. Adhikari NKJ, Tansey MC, McAndrews MP, et al. Self-reported Depressive Symptoms and Memory Complaints in Survivors Five Years After ARDS. CHEST. 2011;140(6):1484-1493.

40. Pearman A. Predictors of Subjective Memory in Young Adults. J Adult Dev. 2009;16:101-107. 